\title{
Pulmonary microbiome patterns correlate with the course of the disease in patients with sepsis- induced ARDS
}

Felix Carl Fabian Schmitt ( $\square$ felix.schmitt@med.uni-heidelberg.de )

Heidelberg University Hospital https://orcid.org/0000-0001-6464-6191

Anna Lipinski

Heidelberg University Hospital

Stefan Hofer

Kaiserslautern Westpfalz Hospital

Florian Uhle

Heidelberg University Hospital

Christian Nusshag

Heidelberg University Hospital

Thilo Hackert

Heidelberg University Hospital

Alexander H. Dalpke

Technical University Dresden

Markus A. Weigand

Heidelberg University Hospital

Thorsten Brenner

Heidelberg University Hospital

Sébastien Boutin

Heidelberg University Hospital

\section{Research article}

Keywords: acute respiratory distress syndrome, sepsis, lung, microbiome, 16S RNA gene sequencing, inflammation, anti-infective therapy

Posted Date: November 7th, 2019

DOI: https://doi.org/10.21203/rs.2.16928/v1

License: (c) (1) This work is licensed under a Creative Commons Attribution 4.0 International License.

Read Full License 


\section{Abstract}

Background Patients with sepsis-induced Acute Respiratory Distress Syndrome (ARDS) are hallmarked by high mortality rates. An early and goal directed antibiotic therapy is crucial for patients' survival. The clinical use of a Next Generation Sequencing (NGS)-based approach for pathogen identification might lead to an improved diagnostic performance. Therefore, the objective of this study was to examine changes in the pulmonary microbiome and resulting influences on patients' outcome in septic ARDS, but also to compare NGS- and culture-based diagnostic methods for pathogen identification.

Results In total, 30 patients in two groups were enrolled in the study: (1) 15 septic ARDS patients and (2) 15 patients undergoing oesophageal resection serving as controls. In the ARDS group, blood samples were collected at ARDS onset as well as 5 days and 10 days afterwards. At the same timepoints, bronchoalveolar lavages (BAL) were performed to collect epithelial lining fluid for culture-, as well as NGSbased analyses and to evaluate longitudinal changes in the pulmonary microbiome. In the control group, only one BAL and one blood sample were collected immediately prior to the surgical procedure. ARDS patients showed a significantly decreased a-diversity $\left(p=0.003^{* *}\right)$ and an increased dominance $\left(p=0.005^{\star *}\right)$ in their pulmonary microbiome. The a-diversity index revealed a good correlation with the length of stay in the intensive care unit $(I C U)(p-v a l u e=0.027)$ and the need for mechanical ventilation ( $p$ value $=0.027$ ). In $42.9 \%$ of all ARDS patients, culture-based results were not concordant with NGS-based findings. Moreover, culture-based results remained negative in 5 cases where NGS-based diagnostics revealed signs of bacterial colonisation.

Conclusion Sepsis-induced ARDS is associated with a significant dysbiosis of patients' pulmonary microbiome, which is closely correlated with the clinical course of the disease. Furthermore, an NGSbased diagnostic approach was shown to be promising for pathogen identification in septic ARDS.

\section{Introduction}

It has been more than 60 years since Acute Respiratory Distress Syndrome (ARDS) was described for the first time [1]. However, it still remains a challenge in modern intensive care medicine. It is hallmarked by a symptom complex, including inflammation, pulmonary oedema due to permeability dysfunctions, diffuse endothelial and epithelial damage and an acute hypoxic-pulmonary failure [2]. The reported incidence of ARDS is about 78/100,000 inhabitants per year [3]. It is either caused by a direct lesion (primary ARDS; e.g. aspiration, lung contusion), or by an indirect lesion (secondary ARDS; e.g. severe sepsis, pancreatitis) [4]. The most frequent and prognostically unfavourable cause for an ARDS is sepsis, amounting for up to $46 \%$ of all cases [5]. Patients with septic ARDS more frequently suffer from a higher disease severity, show an increased 60-day-mortality, require longer periods of mechanical ventilation and undergo a longer ICU stay as compared to patients with an ARDS due to other causes [6].

In the clinical course of ARDS pathophysiological changes in the lung can be frequently observed. A misbalance between pathogen migration and elimination in the respiratory tract, as well as changing 
conditions for microbial reproduction and growth, are the main factors [7]. It has been reported that the pulmonary microbiome changes significantly in the course of various diseases (e.g. chronic obstructive lung disease (COPD)) [8]. If, and to what extent, these results can also be applied to septic ARDS or whether changes in the pulmonary microbiome have an impact on patients' outcome is not fully clarified. Furthermore, we don't know yet whether routinely used culture-based methods have the potential to detect these changes in order to make the right therapeutic decisions according an anti-infective therapy. Therefore, the objectives of this study were to examine changes in the pulmonary microbiome in septic ARDS and its influence on patients' outcome, and also to compare NGS- and culture-based diagnostic methods for pathogen identification.

\section{Results}

\subsection{Patient characteristics}

In total, 15 patients with septic ARDS and 15 patients undergoing oesophageal resection, serving as controls, were enrolled in the present clinical prospective study. Due to technical reasons, the BAL sample volume of one patient in the ARDS group was too small, and therefore not utilisable. Therefore, this patient had to be excluded from the study. ARDS patients suffered from a moderate $(n=4,28.6 \%)$ or severe ARDS ( $n=10,71.4 \%)$ at the time of study enrolment. Sepsis was due to a pulmonary focus in 6 patients $(42.9 \%)$ and 8 patients $(57.1 \%)$ suffered from abdominal sepsis. Control patients revealed a lower American Society of Anaesthesiologists (ASA) status as compared to patients with septic ARDS. Most baseline characteristics, such as age or gender, did not differ significantly between both groups (Table 1). However, ARDS patients showed significantly increased plasma levels of C-reactive protein (CRP) and a higher leucocyte count as compared to control patients at $\mathrm{dd}$ (Figure 1A, 1B). Moreover, the length of the ICU stays and the need for mechanical ventilation was prolonged in ARDS patients. Two control patients ( $\mathrm{C} 2 \& \mathrm{C} 7$ ) suffered from severe pneumonia during the first week following oesophageal resection, subsequently resulting in septic ARDS in both cases. Therefore, both patients could also be enrolled in the ARDS group (A2 \& A7). Another two control patients (C8 \& C10) also suffered from pneumonia within the first days after oesophageal resection but without fulfilling sepsis or ARDS criteria. All 15 patients in the control and 10 patients $(71.4 \%)$ in the ARDS group survived the 180-day observation period.

\subsection{Microbiome results}

On the day of admission, patients in the ARDS group were characterised by a significantly decreased adiversity $\left(p=0.003^{\star \star}\right)$ and an increased dominance $\left(p=0.005^{\star \star}\right)$ in the BAL (Figure $\left.1 B, 1 C\right)$ compared to the control group. Principle coordinates analysis (PCOA) clearly distinguished between ARDS and control patients (Figure 2), , representing a strong indicator for profound changes in the microbiome's structure, as validated by PERMANOVA $(p=0.002 * \star)$. Axis 1 discriminates best between ARDS and control patients, which is negatively correlated with bacteria considered as constituents of the physiological lung flora 
(Streptococcus, Haemophilus, Neisseria) and mostly anaerobic bacteria (Prevotella, Veillonella, Capnocytophaga) (Suppl. Figure 1).. This is in line with the results from differential abundance analyses (log limma-2 method), which highlight a general decrease in bacteria belonging to the physiological lung flora in ARDS patients (Table 2).

In ARDS patients, the relation between changes in the microbiome and clinical parameters was evaluated. The a-diversity index showed a good correlation with several clinical parameters, such as the length of stay in the intensive care unit $(I C U)(p$-value $=0.027)$ and the need for mechanical ventilation $(p$-value $=$ 0.027) (Figure 3)..

ARDS patients revealed a high heterogeneity in their microbiome and most patients had a high dominance of one specific ribosomal sequence variants (RSV) (>30\%). Due to the high variability of the dominating RSV, no significant associations between a specific RSV and clinical parameters were observed.

\subsection{Next Generation Sequencing (NGS)- vs. culture-based methods for pathogen identification}

In $42.9 \%$ of ARDS patients, culture-based results were not concordant with NGS-based microbiota results. Furthermore, 5 of 14 culture-based results were negative, even though NGS showed a bacterial colonisation (Table 3). Patients A8, A11, A14 as well as A15 deceased within the 180-day observation period. All these patients were hallmarked by a mismatch of culture- and NGS-based results already at $0 \mathrm{~d}$ $(A 11, A 14, A 15)$ or at later stages (A8). For example, in patient A8 culture-based methods failed to detect Pseudomonas spp. at d5 and d10 (Figure 4)..

\subsection{Subgroup analyses}

In seven ARDS patients, a pre-existing pulmonary disease was recorded. Nevertheless, no significant differences were observed with regard to a-diversity $(p=0.128)$, dominance $(p=0.456)$, richness $(p=$ $0.403)$ or evenness $(p=1)$ between ARDS patients with and without a pre-existing pulmonary disease. Six patients $(42.9 \%)$ suffered from a pneumonia, whereas an abdominal focus due to anastomosis insufficiency, bowel ischaemia, pancreatitis or intraabdominal abscess formations was present in 8 patients $(57.1 \%)$. In patients with a pulmonary focus, the microbiome showed a significantly different structure at the phylum level as compared to patients with an abdominal focus $\left(p=0.008^{\star \star}\right)$. This difference was due to a richer microbiome in the cohort of patients with a pneumonia $\left(p=0.027^{\star}\right)$. Moreover, the phylum Firmicutes (log-limma2 test: $\log F C=3.098$, q-value $=0.035$ ) and Bacteroidetesloglimma2 test: $\log F C=3.609$, q-value $=0.024$ ) were more abundant in patients with a pulmonary focus, whereas Proteobacteria were more abundant in patients with an abdominal focus (log-limma2 test: logFC $=-1.841$, q-value $=0.024)$. However, the differences between the groups were not statistically significant at the RSV level. 


\section{Discussion}

ARDS can be caused by a variety of direct (e.g. aspiration, lung contusion) or indirect causes (e.g. sepsis, pancreatitis) [11] and, until now, has been associated with high mortality rates $[3,9,10]$. However, severity and mortality rates are particularly devastating in patients with sepsis-induced ARDS [6]. Several studies have shown that the gastrointestinal microbiome changes drastically in critical illness [12-15]. A similar effect on the pulmonary microbiome could be observed in septic ARDS patients within the present work. Already, at the onset of ARDS, a significant dysbiosis could be observed, corresponding with a highly diminishing diversity and a clear difference between the ARDS and control groups. ARDS patients showed a significant decrease in diversity and an overgrowth of one specific RSV, whereas control patients revealed a physiological pulmonary flora. ARDS-associated changes and remodelling processes aggravate this significant decrease in bacterial diversity, together with a flare-up of problematic bacteria mostly belonging to the Proteobacteria phylum such as Pseudomonas aeruginosa [16]. Because of harmful direct and indirect noxious agents, the alveolo-capillary membrane function could be hampered with a migration of neutrophil granulocytes $[17,18]$. Even though macrophages and neutrophilic granulocytes play an important role regarding resistance towards pathogens, they can trigger cytokinemediated apoptosis, inducing necrosis in pneumocytes [19]. The fatal cycle of cell activation and epithelial damage results in an elevated permeability and a loss of epithelial barrier function, inevitably leading to a transfer of further inflammatory mediators and pathogens [20]. Not surprisingly, the physiological composition of the pulmonary microbiome is compromised and mono-specific infection with high pathogenic relevance do occur. This effect could impressively be shown in patients C2 and C7 of the control group, suffering from septic ARDS following surgery. Therefore, these patients were also included in the ARDS group (A2 and A7). At study inclusion prior to oesophageal resection, both patients showed a distinct similarity on the PCoA as compared to the remaining control patients. However, the pulmonary microbiome of both patients clearly shifted to the right side of the PCOA at ARDS onset, as is the case in all other ARDS patients. Furthermore, two other control patients (C8 and C10) suffered from pneumonia within the first days following oesophageal resection, but without resulting in sepsis and/or ARDS. Interestingly, these two patients revealed a remarkably modified pulmonary microbiome already prior to surgery, rather resembling the rest of the ARDS patients. Both patients did not show any clinical signs for an infection at the day of oesophageal resection, otherwise the operation would have been postponed. However, microbiome analyses were able to detect the emerging complicated course already at $0 \mathrm{~d}$, much earlier than any clinical signs for an upcoming infection could be observed.

Differences in the individual pulmonary microbiome could be affected by pre-existing pulmonary diseases. In particular, patients with chronic lung diseases such as COPD or asthma seem to be affected [7]. An increased production of mucus and the corresponding high availability of nutrient-rich substrates, an improved temperature setting and a modified concentration of oxygen seem to support the occurrence of new dominant species in the pulmonary microbiome [8]. Surprisingly, this effect could not be observed within the presented work, revealing a comparable alpha-diversity, dominance, richness and evenness in the pulmonary microbiome of ARDS patients with a pre-existing lung disease as compared to ARDS patients without any lung diseases. On the contrary, differences in the composition of the pulmonary 
microbiome could be observed depending on the ARDS cause. In patients suffering from a pneumonia, Firmicutes and Bacteroidetes phylumcould be detectedmore frequently, potentially representing a transient or an intermediate state of the hampered microbiome. This is due to the fact that patients with a pulmonary focus are characterised by moderate dysbiosis, in between the highly dysbiotic patients with an abdominal focus and the physiological microbiome observed in patients of the healthy control group. In line with this, patients with a pulmonary focus had a shorter ICU stay and were less dependent on mechanical ventilation, as compared to patients with an abdominal focus.

Regardless of the underlying ARDS cause, early and targeted antibiotic therapy is crucial for patients' survival. For the correct antibiotic choice, a reliable and fast microbiological diagnostic approach is required. Unfortunately, currently used culture-based techniques are associated with relevant weaknesses: culture-based diagnostic procedures do not only require several days for test results to become available but, in addition, the rate of false-negative test results is still unacceptably high (30-40\%). This also holds true for cases in which clinical symptoms are highly suggestive for an underlying infection [21].Reasons for this high rate of false negative test results could include (1.) a low density of bacteria in the lungs, (2.) difficulties in cultivating distinct bacterial strains or (3.) an ongoing antibiotic therapy. However, false positive results due to a contamination of the sample with bacteria from the upper respiratory tract might also affect therapeutic decisions [22-25]. Without any doubt, most of these limitations might also apply for NGS-based techniques. However, an NGS-based approach was able to detect changes in the pulmonary microbiome of control patients prior to the occurrence of the first clinical symptoms. Moreover, culture and NGS-based diagnostic procedures revealed discordant results in more than half of ARDS patients. Deceased patients with septic ARDS $(n=4)$ were affected by a corresponding inaccuracy. However, this inaccuracy is not inevitably linked with the need for a therapeutic intervention (e.g. colonisation with Stenotrophomonas spp.). Nevertheless, culture-based diagnostic procedures also failed to detect clinically relevant pathogens, such as Pseudomonas spp. in patient A8. To date, these inaccuracies have been at least partially responsible for the excessive use of broad-spectrum antibiotics and a delayed de-escalation strategy due to safety reasons [26]. This leads to the question, whether the use of an NGS based approach in daily clinical practice would result in a higher diagnostic accuracy and a more targeted therapy. The suitability of these new techniques has already been proven successfully during the Ebola epidemic in West-Africa [27].

This study is a single-centre study with a restricted number of included cases. Therefore, in some points statistically reliable conclusions cannot be made. The BALs were performed only on one spot in the area of the right lower lobe. It cannot be excluded that the pathogen load in other lung areas was under- or overestimated. Even though the alpha-diversity correlates with clinical parameters, such as the length of ICU stay and the duration of mechanical ventilation, the exact impact of the microbiome on the disease course is yet to be determined.

\section{Conclusion}


Patients with septic ARDS showed a significant dysbiosis already at the onset of ARDS as compared to the control group. Changes in the pulmonary microbiome revealed a good correlation with the patients' disease course. Furthermore, depending on the underlying cause of the ARDS (pulmonary vs. abdominal) profound differences in the composition of the pulmonary microbiome could be observed in ARDS patients. Alarmingly, conventional culture-based methods often remained falsely negative. Therefore, an NGS-based diagnostic approach might be promising for future clinical practice.

\section{Materials And Methods \\ 5.1 Study design}

This prospective, observational clinical study was approved by the Ethics Committee of the Medical Faculty of Heidelberg (trial code no. S-063/2015) and was prospectively registered in the German clinical trials register (DRKS-ID: DRKS00008317). All study patients or their legal representatives signed written informed consent. Between November 2015 and November 2016, 30 patients in two groups were enrolled in the study: (1.) 15 septic patients with an ARDS according to the Berlin definition [28] and (2.) 15 patients undergoing oesophageal resection without any pre-existing pulmonary diseases as the designated control group. The following criteria were defined as exclusion criteria: pregnancy or patients under the age of 18 years. ARDS patients were re-evaluated for survival 180 days after enrolment in the study. Relevant baseline data were collected at the time of study inclusion in both groups. In patients suffering from ARDS, blood samples were collected at ARDS onset ( $0 d$ ) as well as at 5 days ( $5 d)$ and 10 days $(10 \mathrm{~d})$ later. At the same times, bronchoalveolar lavages (BAL) were performed in order to collect epithelial lining fluid for culture-based analyses in the central lab of the Department of Infectious Diseases, Medical Microbiology and Hygiene at the University Hospital Heidelberg. Additionally, further BAL samples were obtained to evaluate longitudinal changes in the pulmonary microbiome. In the control group, BAL and blood samples were collected once, prior to the surgical procedure, in order to determine the baseline microbiome as well as the laboratory standard parameters without surgical stress or antibiotic usage $(0 \mathrm{~d})$.

\subsection{Collection and storage of BAL samples}

All BALs were performed under sterile conditions. For microbiome analyses, $2 \times 50 \mathrm{ml}$ of sodium chloride $0.9 \%$ were injected in the right lower lobe. The first $50 \mathrm{ml}$ were discarded; the second $50 \mathrm{ml}$ were put in a sterile vacuum-system and then snap-frozen in liquid nitrogen. Afterwards the samples were stored at $80^{\circ} \mathrm{C}$ until further processing.

DNA extractions were performed using the QIAamp Mini Kit (QIAGEN, Hilden, Germany). Protease solution (7.2 $\mathrm{mAU})$ and $200 \mu \mathrm{L}$ of buffer AL were added to the sample followed by a $15 \mathrm{sec}$ vortex. Samples were incubated at $56^{\circ} \mathrm{C}$ for $10 \mathrm{~min}$ and then purified according to the manufacturer's protocol. DNA was eluted by adding $100 \mu \mathrm{L}$ of buffer $A E$ to the test tube, incubation for $1 \mathrm{~min}$ at room temperature and a centrifugation at $6000 \mathrm{xg}$ for $1 \mathrm{~min}$. Negative controls were performed by doing the extraction without 
clinical samples to exclude any contamination due to the process itself. DNA was amplified using universal bacterial primers flanking the V4 region (515F and 806R). Each primer was tagged with an individual barcode (each barcode had at least $3 \mathrm{nt}$ differences to the others) to assign the sequences to the samples. PCR reactions were performed in $25 \mu \mathrm{L}$ volumes composed of Q5 High-Fidelity 1X Master Mix (New England Biolabs $\mathrm{GmbH}$, Germany), 25 pmol of each primer and $2 \mu \mathrm{L}$ of DNA. The thermal cycling (Primus 25, Peqlab Biotechnologie GmbH, Germany or FlexCycler², Analytik Jena AG, Germany) conditions were: A first denaturation at $94^{\circ} \mathrm{C}$ for $3 \mathrm{~min}, 30$ amplification cycles $\left(94^{\circ} \mathrm{C}\right.$ for $45 \mathrm{sec}, 50^{\circ} \mathrm{C}$ for $1 \mathrm{~min}$ and $72^{\circ} \mathrm{C}$ for $1 \mathrm{~min} 30 \mathrm{sec}$ ) and a final extended cycle at $72^{\circ} \mathrm{C}$ for 10 minutes. PCR products were checked by agarose gel electrophoresis $(2 \%)$ for the presence of amplicons. PCR was also performed with negative controls from the extraction as well as with sterile water to exclude contamination from the extraction kit and the PCR master mix. Both negative controls were negative on the gel. An internal control was performed by amplifying a mock community sample containing genomic DNA from 20 bacterial strains in equimolar (even) ribosomal RNA operon counts (HMD-782D, BEl resources, ATCC, USA). PCR products were then purified with magnetic beads MagSi-NGS prep Plus (Steinbrenner Laborsystem GmBH, Wiesenbach, Germany). Purified PCR products were quantified using the Quant-iT ${ }^{\mathrm{TM}}$ PicoGreen $^{\mathrm{TM}} \mathrm{dsDNA}$ Assay Kit (ThermoFisher scientific, Germany) and an ND-1000 Nanodrop instrument (Nanodrop, Wilmington, USA). Samples were pooled as an equimolar mix and sent to GATC (Konstanz, Germany) to perform the ligation to the sequencing adapters and paired-end sequences on an Illumina Miseq system (250 cycles). In one BAL sample the analysis failed due to a low PCR output.

\subsection{Microbiome analysis}

Raw sequences were analysed with the $\mathrm{R}$ package dada2. Raw sequences were filtered and trimmed with the following parameters: maximum ambiguity: 0 , number of expected errors for each read: 1 , truncate reads at the first instance of a quality score less than 2 . Reads were then merged as contigs and checked for chimera with the default parameters. RSV were then assigned to taxonomy using the Silva database (version 132). RSV associated with eukaryotes, archaea and chloroplasts were removed from the analysis. The RSV table was used to calculate descriptive indices for alpha-diversity (non-parametric Shannon index), richness (number of RSV observed) and dominance (relative abundance of the most dominant RSV). 1,469,880 reads in total were used with a mean number of 24,913 reads per samples. Rarefaction curves for all the samples were evaluated to check if a plateau was reached.

\subsection{Statistical analysis}

Beta-diversity measures were performed to examine the differences between the samples based on Morisita-horn distances. The search for differences in the structure of the microbiome of the groups was performed by using PERMANOVA with the co-variable age taken in account. Diversity index comparisons between groups were performed with a Mann-Whitney test to consider a non-Gaussian distribution. To compare microbial changes at the RSV levels, we used the package DAtest to evaluate which model would be the most accurate model for our study (based on AUC, FDR and FPR) and the log-limma 2 was 
determined to be the best model for our study. A check for correlations between the microbiome data and the clinical parameters was performed with a Spearman correlation test, adjusted for multiple comparisons. All statistical analyses were performed with R 3.4.4 and the packages DAtest, microbiome and phyloseq.

The clinical data were entered into an electronic database (Excel 2017, Microsoft Corp., Redmond, WA, USA) and analysed using SPSS software (version 24.0; SPSS Inc., Chicago, IL, USA). Categorical data were summarised using absolute and relative frequencies. Quantitative data were summarised using the median and quartiles. The Kolmogorov-Smirnov test was applied to check for a normal distribution. In the case of non-normally distributed data, non-parametric methods were used for evaluation (chi-square test for categorical data, Mann-Whitney $U$ test for continuous data). Correlation analyses were performed by calculating the Spearman rank correlation coefficient (Spearman's rho/(ם). A p-value of $<0.05$ was considered statistically significant. The following symbols were used to represent higher orders of significance, $p<0.05$ was indicated by $*, p<0.01$ by ** and $p<0.001$ by $* \star *$.

\section{Declarations \\ Ethics approval}

This prospective, observational, clinical study was approved by the local ethics committee (Ethics Committee of the Medical Faculty of Heidelberg; Trial-Code No. S-063/2015; German clinical trials register DRKS00008317). All study patients or their legal representatives signed written informed consent.

\section{Consent for publication}

Not applicable.

\section{Availability of data and materials}

The detailed datasets generated and analysed during the current study are not publicly available due to federal patient privacy regulations but are available from the corresponding author on reasonable request.

\section{Competing interests}

The authors declare that the research was conducted in the absence of any commercial or financial relationships that could be construed as a potential conflict of interest.

\section{Funding}


This study was carried out with the financial resources of the Department of Anaesthesiology, the Department of General and Transplant Surgery and the Department of Infectious Diseases, Medical Microbiology and Hygiene at Heidelberg University Hospital, Heidelberg, Germany. Further support was provided by German Ministry for Education and Research (82DZL00401, 82DZL004A1).

\section{Author's specific contributions}

FS, TB, SH, MW and SB conceived the study and participated in its design. $F S, T B, F U, A L, T H, C N, S H, A D$, $\mathrm{MW}$ and $\mathrm{SB}$ acquired and analysed the study data. $\mathrm{FU}, \mathrm{AL}, \mathrm{TH}, \mathrm{CN}$ and $\mathrm{AD}$ participated substantially in the organisation and coordination of the study. FS and SB wrote the manuscript and prepared the tables and figures. All authors reviewed and revised the manuscript critically.

\section{Acknowledgements}

The authors thank S. Hassel for her excellent technical assistance

\section{References}

1.Ashbaugh DG, et al., Acute respiratory distress in adults. Lancet. 1967;2(7511):319-23.

2.Koh, Y., Update in acute respiratory distress syndrome. J Intensive Care. 2014;2(1):2.

3.Rubenfeld GD, et al., Incidence and outcomes of acute lung injury. N Engl J Med. 2005;353(16):168593.

4.Luo L, et al., Clinical Predictors of Hospital Mortality Differ Between Direct and Indirect ARDS. Chest. 2017;151(4):755-63.

5.Modrykamien AM and Gupta P, The acute respiratory distress syndrome. Proc (Bayl Univ Med Cent). 2015;28(2):163-71.

6.Sheu CC, et al., Clinical characteristics and outcomes of sepsis-related vs non-sepsis-related ARDS. Chest. 2010;138(3):559-67.

7.Dickson RP, The microbiome and critical illness. Lancet Respir Med, 2016. 4(1):59-72.

8. Huffnagle GB and Dickson RP, The bacterial microbiota in inflammatory lung diseases. Clin Immunol. 2015;159(2):177-82.

9.Bellani G, et al., Epidemiology, Patterns of Care, and Mortality for Patients With Acute Respiratory Distress Syndrome in Intensive Care Units in 50 Countries. JAMA. 2016;315(8): 788-800. 
10.Villar J, Schultz MJ, and Kacmarek RM, The LUNG SAFE: a biased presentation of the prevalence of ARDS! Crit Care. 2016;20(1):108.

11.Bernard GR, et al., The American-European Consensus Conference on ARDS. Definitions, mechanisms, relevant outcomes, and clinical trial coordination. Am J Respir Crit Care Med. 1994;149(3 Pt 1):818-24.

12.Lankelma JM, et al., Critically ill patients demonstrate large interpersonal variation in intestinal microbiota dysregulation: a pilot study. Intensive Care Med. 2017;43(1):59-68.

13.McDonald D, et al., Extreme Dysbiosis of the Microbiome in Critical IIIness. mSphere. 2016; 1(4).

14.0jima M, et al., Metagenomic Analysis Reveals Dynamic Changes of Whole Gut Microbiota in the Acute Phase of Intensive Care Unit Patients. Dig Dis Sci. 2016;61(6):1628-34.

15.Zaborin A, et al., Membership and behavior of ultra-low-diversity pathogen communities present in the gut of humans during prolonged critical illness. MBio. 2014;5(5):e01361-14.

16.Matthay MA, Ware LB, and Zimmerman GA, The acute respiratory distress syndrome. J Clin Invest. 2012;122(8):2731-40.

17.Morisawa K, et al., Difference in pulmonary permeability between indirect and direct acute respiratory distress syndrome assessed by the transpulmonary thermodilution technique: a prospective, observational, multi-institutional study. J Intensive Care. 2014;2(1):24.

18.Ware LB and Matthay MA, The acute respiratory distress syndrome. N Engl J Med. 2000;342(18):1334-49.

19.Williams AE and Chambers RC, The mercurial nature of neutrophils: still an enigma in ARDS? Am J Physiol Lung Cell Mol Physiol. 2014;306(3):L217-30.

20.Menezes SL, et al., Pulmonary and extrapulmonary acute lung injury: inflammatory and ultrastructural analyses. J Appl Physiol (1985). 2005;98(5):1777-83.

21.Esperatti $\mathrm{M}$, et al., Nosocomial pneumonia in the intensive care unit acquired by mechanically ventilated versus nonventilated patients. Am J Respir Crit Care Med. 2010;182(12):1533-9.

22.Segal LN and Blaser MJ, A brave new world: the lung microbiota in an era of change. Ann Am Thorac Soc. 2014;11 Suppl 1:S21-7.

23.Browne HP, et al., Culturing of 'unculturable' human microbiota reveals novel taxa and extensive sporulation. Nature. 2016;533(7604):543-6.

24.Venkataraman A, et al., Application of a neutral community model to assess structuring of the human lung microbiome. MBio. 2015;6(1). 
25.Kitsios GD, et al., Dysbiosis in the intensive care unit: Microbiome science coming to the bedside. J Crit Care. 2017;38:84-91.

26.Kitsios GD, Morris A, and McVerry BJ, Antibiotic de-escalation: observational causal inference and culture dependence. Intensive Care Med. 2016;42(10):1647-8.

27.Quick J, et al., Real-time, portable genome sequencing for Ebola surveillance. Nature.

2016;530(7589):228-32.

28.Force ADT, et al., Acute respiratory distress syndrome: the Berlin Definition. JAMA.

2012;307(23):2526-33.

\section{Tables}

Table 1: Patients' characteristics.

\begin{tabular}{|c|c|}
\hline \multicolumn{2}{|l|}{ Surgical control group } \\
\hline Age [years] & $59(51.00-64.50)$ \\
\hline Gender [male] & $12(80.00 \%)$ \\
\hline ASA status I; II; III; IV; V & $1(6.67 \%) ; 7(46.67 \%) ; 7$ (46.67\%); 0 (0.00\%); 0 (0.00\%) \\
\hline \multicolumn{2}{|l|}{ Surgical site } \\
\hline Oesophagus & $15(100.00 \%)$ \\
\hline \multicolumn{2}{|l|}{ ARDS group } \\
\hline Age [years] & $59.0(53.25-70.75)$ \\
\hline Gender [male] & $13(92.86 \%)$ \\
\hline ASA status I; II; III; IV; V & 1 (7.14\%); 1 (7.14\%); $11(78.57 \%) ; 1$ (7.14\%); 0 (0.00\%) \\
\hline \multicolumn{2}{|l|}{ Pulmonary diseases } \\
\hline$C O P D$ & $5(35.71 \%)$ \\
\hline Asthma & $1(7.14 \%)$ \\
\hline Recurrent pulmonary infections & $1(7.14 \%)$ \\
\hline \multicolumn{2}{|l|}{ Surgical site } \\
\hline Gastrointestinal tract & $5(35.71 \%)$ \\
\hline Liver & $3(21.43 \%)$ \\
\hline Oesophagus & $3(21.43 \%)$ \\
\hline Genito-urinary tract & $2(14.29 \%)$ \\
\hline Others & $1(7.14 \%)$ \\
\hline \multicolumn{2}{|l|}{ Septic focus } \\
\hline Pulmonary & $6(42.86 \%)$ \\
\hline Abdominal & $8(57.14 \%)$ \\
\hline \multicolumn{2}{|l|}{ Hospital stay } \\
\hline Intensive care unit [days] & $19.00(12.50-33.50)$ \\
\hline Intermediate care unit [days] & $10.00(5.00-14.00)$ \\
\hline Need for mechanical ventilation [hours] & $564.00(264.00-780.00)$ \\
\hline \multicolumn{2}{|l|}{ Outcome } \\
\hline Survivor 180 days & $10(71.43 \%)$ \\
\hline
\end{tabular}


Table 2: Differentially abundant RSVs between control and ARDS patients. The differential abundance was tested with the log limma2 method and the p-value was adjusted using the FDR method.

\begin{tabular}{|c|c|c|c|c|c|}
\hline RSV & Taxonomy & Ordering & FC & $\log _{2} \mathrm{FC}$ & p-value (adj.) \\
\hline RSV 2 & Haemophilus & Control > ARDS & 5.300 & 2.406 & 0.004 \\
\hline RSV 2216 & Veillonella & Control > ARDS & 6.097 & 2.608 & $<0.001$ \\
\hline RSV 834 & Veillonella & Control > ARDS & 1.594 & 0.673 & 0.048 \\
\hline RSV 2321 & Prevotella melaninogenica & Control > ARDS & 4.704 & 2.234 & 0.002 \\
\hline RSV 2748 & Prevotella histicola & Control > ARDS & 3.299 & 1.722 & 0.013 \\
\hline RSV 5198 & Prevotella salivae & Control > ARDS & 2.518 & 1.332 & 0.034 \\
\hline RSV 425 & Prevotella nanceiensis & Control > ARDS & 1.815 & 0.860 & 0.030 \\
\hline RSV 5285 & Alloprevotella & Control > ARDS & 2.372 & 1.246 & 0.034 \\
\hline RSV 5080 & Campylobacter concisus & Control > ARDS & 3.683 & 1.881 & $<0.001$ \\
\hline RSV 3375 & Gemella & Control > ARDS & 3.635 & 1.862 & 0.002 \\
\hline RSV 5231 & Megasphaera micronuciformis & Control > ARDS & 2.693 & 1.429 & 0.008 \\
\hline RSV 5371 & Porphyromonas pasteri & Control > ARDS & 2.291 & 1.196 & 0.042 \\
\hline
\end{tabular}

Significance levels are indicated as follows: $p<0.05: *, p<0.01: \star \star, p<0.001$ : $* \star \star$ Abbreviations: RSV, ribosomal sequence variants; ARDS, acute respiratory distress syndrome; FC, fold change;

Table 3: Results of the NGS- and culture-dependent methods at d0. 


\begin{tabular}{|c|c|c|}
\hline $\begin{array}{l}\text { Study } \\
\text { Participant }\end{array}$ & NGS-based diagnostics & Culture-dependent methods \\
\hline A1 & $\begin{array}{l}\text { Streptococcus, Prevotella, Veillonella, Flavobacterium, Porphyromonas, } \\
\text { Fusobacterium, Gemella, Alloprevotella }\end{array}$ & Streptococcus viridans \\
\hline A2 & $\begin{array}{l}\text { Streptococcus, Neisseria, Capnocytophaga, Fusobacterium, Mycoplasma, } \\
\text { Alloprevotella, Haemophilus, Prevotella, Porphyromonas }\end{array}$ & $\begin{array}{l}\text { Streptococcus viridans, } \\
\text { Neisseria }\end{array}$ \\
\hline A3 & Pseudomonas, Flavobacterium & Pseudomonas aeruginosa \\
\hline A4 & Pseudomonas, Streptococcus, Flavobacterium, Lactobacillus & Pseudomonas aeruginosa \\
\hline A5 & Serratia, Streptococcus, Achromobacter, Delftia & Serratia marcescens \\
\hline A6 & Streptococcus, Delftia, Corynebacterium, Achromobacter, Staphylococcus & BAL negative \\
\hline A7 & $\begin{array}{l}\text { Staphylococcus, Streptococcus, Prevotella, Haemophilus, Veilonella, Neisseria, } \\
\text { Fusobacterium }\end{array}$ & $\begin{array}{l}\text { Staphylococcus aureus, } \\
\text { Streptococcus viridans }\end{array}$ \\
\hline A8 & Pseudomonas & Pseudomonas aeruginosa \\
\hline A10 & $\begin{array}{l}\text { Enhydrobacter, Stenotrophomonas, Staphylococcus, Streptococcus, Haemophilus, } \\
\text { Lactobacillus, }\end{array}$ & BAL negative \\
\hline A11 & Enterococcus, Acinetobacter, Delftia, Corynebacterium & $\begin{array}{l}\text { Streptococcus viridans, non- } \\
\text { haemolytic streptococci }\end{array}$ \\
\hline A12 & E.coli, Lactobacillus, Staphylococcus, Flavobacterium & E.coli \\
\hline A13 & Cutibacterium, Paracoccus, Delftia & BAL negative \\
\hline A14 & Paracoccus, Achromobacter, Delftia & BAL negative \\
\hline A15 & Stenotrophomonas, Corynebacterium, Sphingomonas, Delftia & BAL negative \\
\hline
\end{tabular}

\section{Figures}



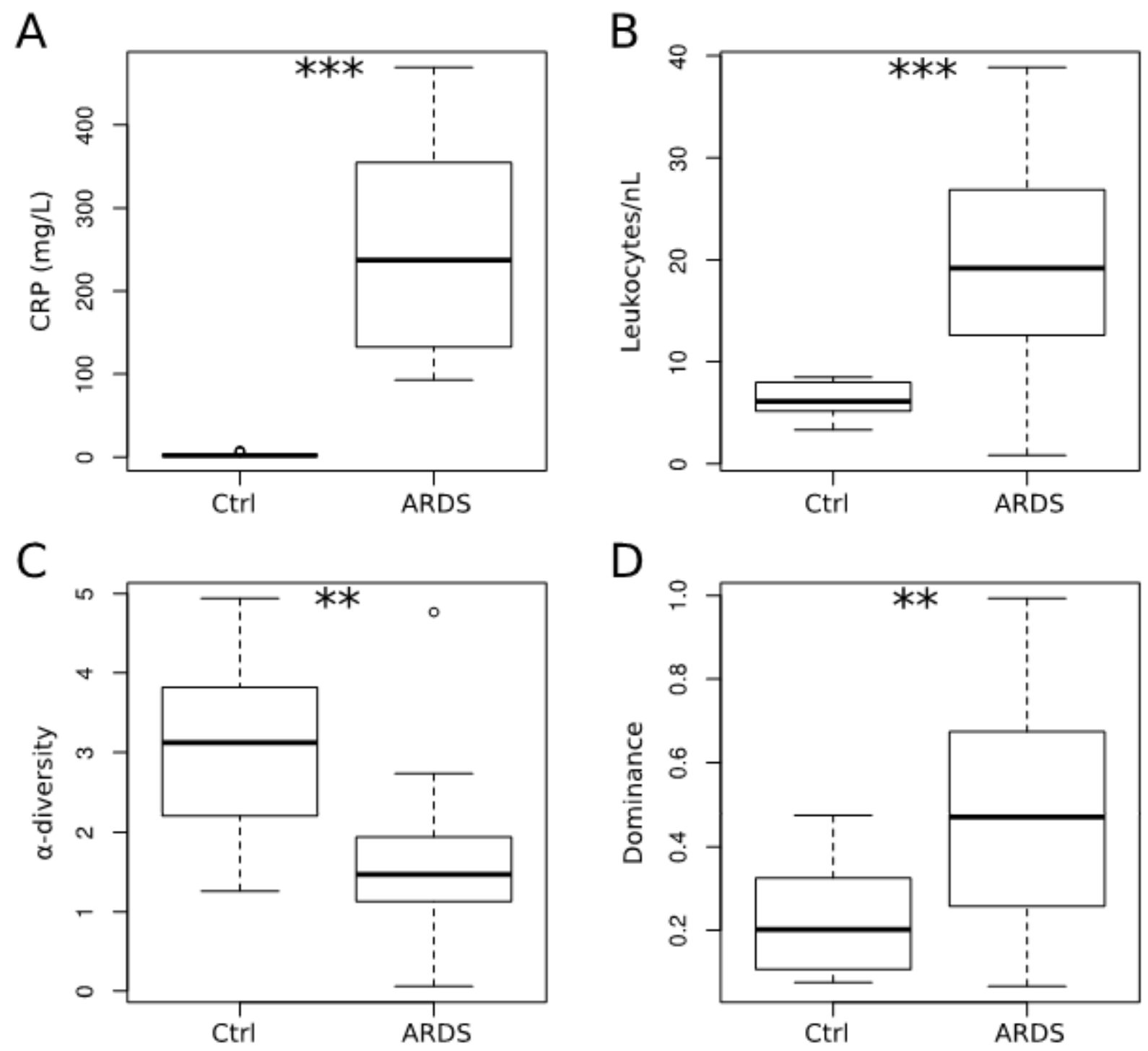

\section{Figure 1}

ARDS patients showed higher CRP and leucocyte concentrations associated with changes in the microbiota's diversity. Plasma levels of CRP and leucocytes at d0. \-diversity was measured using Shannon index and dominance is estimated as the relative abundance of the most abundant RSV. Statistical difference was evaluated using the Mann-Whitney test. Significance levels are indicated as follows: $p<0.05:{ }^{*}, p<0.01: * \star, p<0.001$ : ${ }^{* \star}$. Abbreviations: ARDS, acute respiratory distress syndrome; CRP, C-reactive protein; RSV, ribosomal sequence variants. 


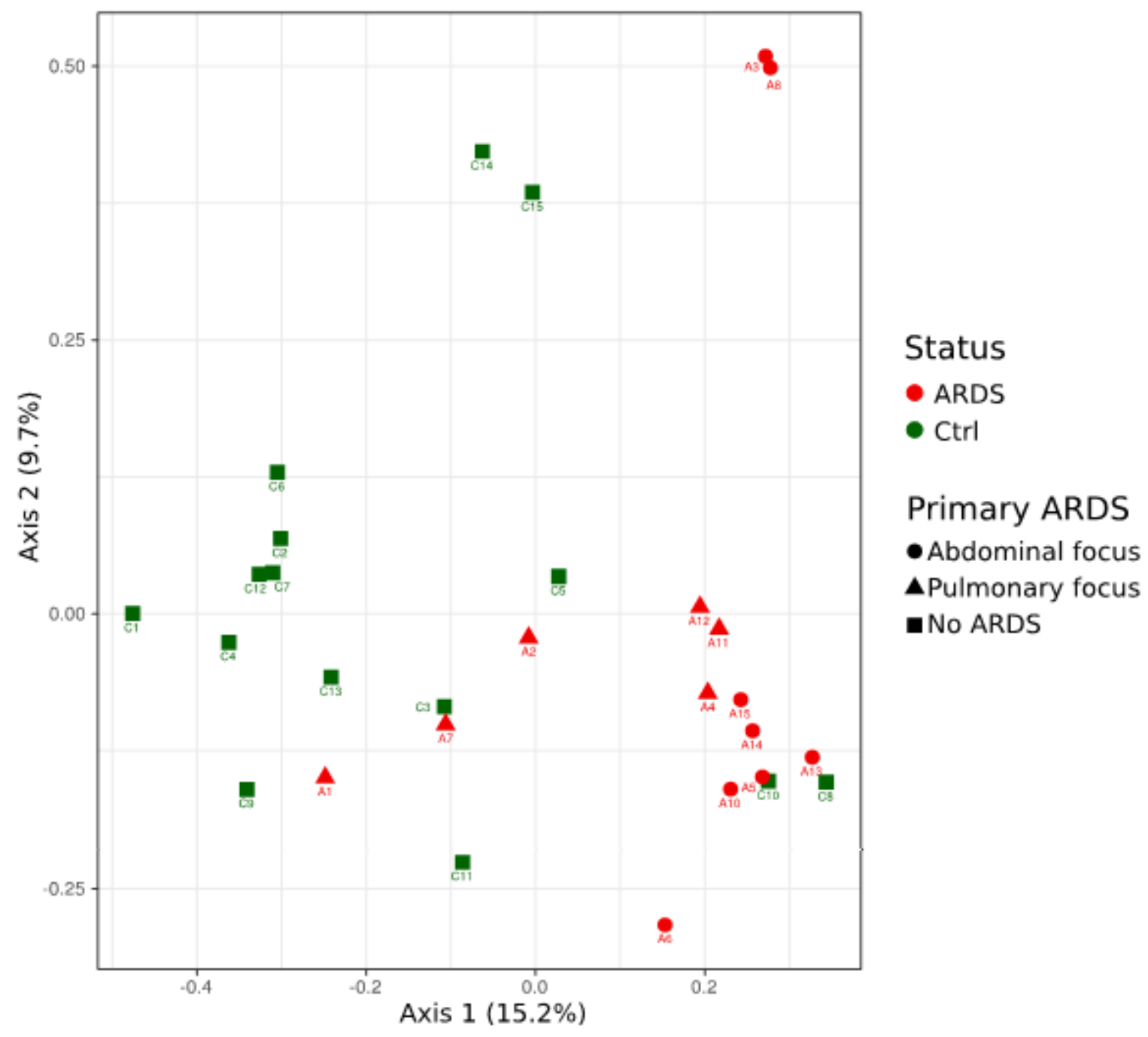

Figure 2

Patients with ARDS showed a different microbiota structure in the lung as compared to controls. PCoA was performed based on Morisita-Horn dissimilarity matrix. Statistical differences between the two groups were evaluated with PERMANOVA including the covariable age in the model $\left(R^{2}=0.07, p-\right.$ value $=0.002)$. Significance levels are indicated as follows: $p<0.05:{ }^{*}, p<0.01: * \star, p<0.001$ : $* \star \star$. Abbreviations: ARDS, acute respiratory distress syndrome; Crtl, Control; PCoA, Principle coordinates analysis. 


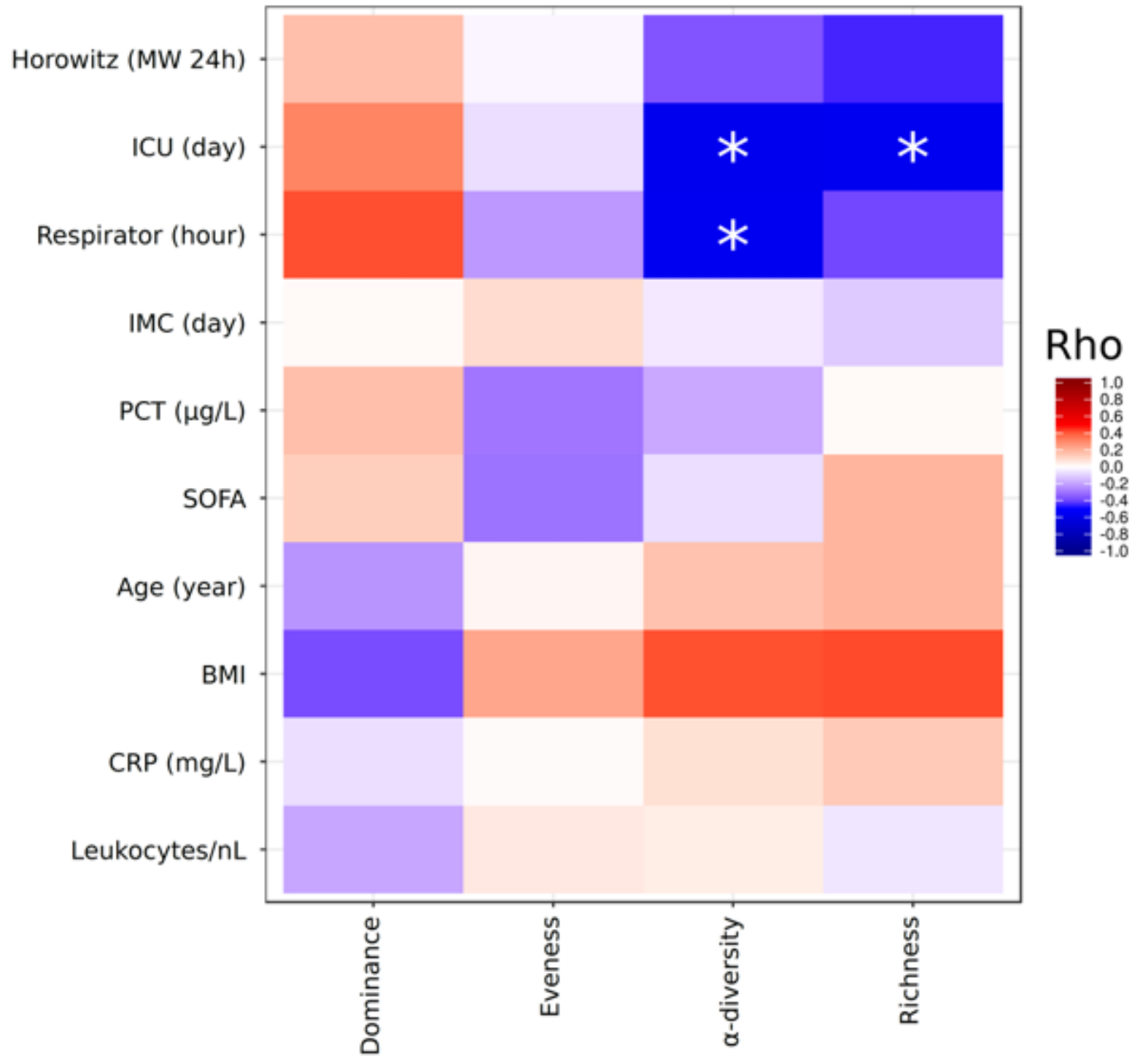

\section{Figure 3}

Microbial diversity correlated with a better outcome in ARDS patients. Correlation between diversity parameters of the microbiota and clinical data were performed using a Spearman rank correlation test. Pvalues were adjusted using the FDR method. Significance levels are indicated as follows: $p<0.05: *, p<$ $0.01:{ }^{*}, \mathrm{p}<0.001:{ }^{* \star}$. Abbreviations: ARDS, acute respiratory distress syndrome; FDR, false discovery rate; ICU, intensive care unit; IMC, intermediate care unit; PCT, Procalcitonin; SOFA, Sepsis-related organ failure assessment (-score); BMI, body mass index; CRP, C-reactive protein. 

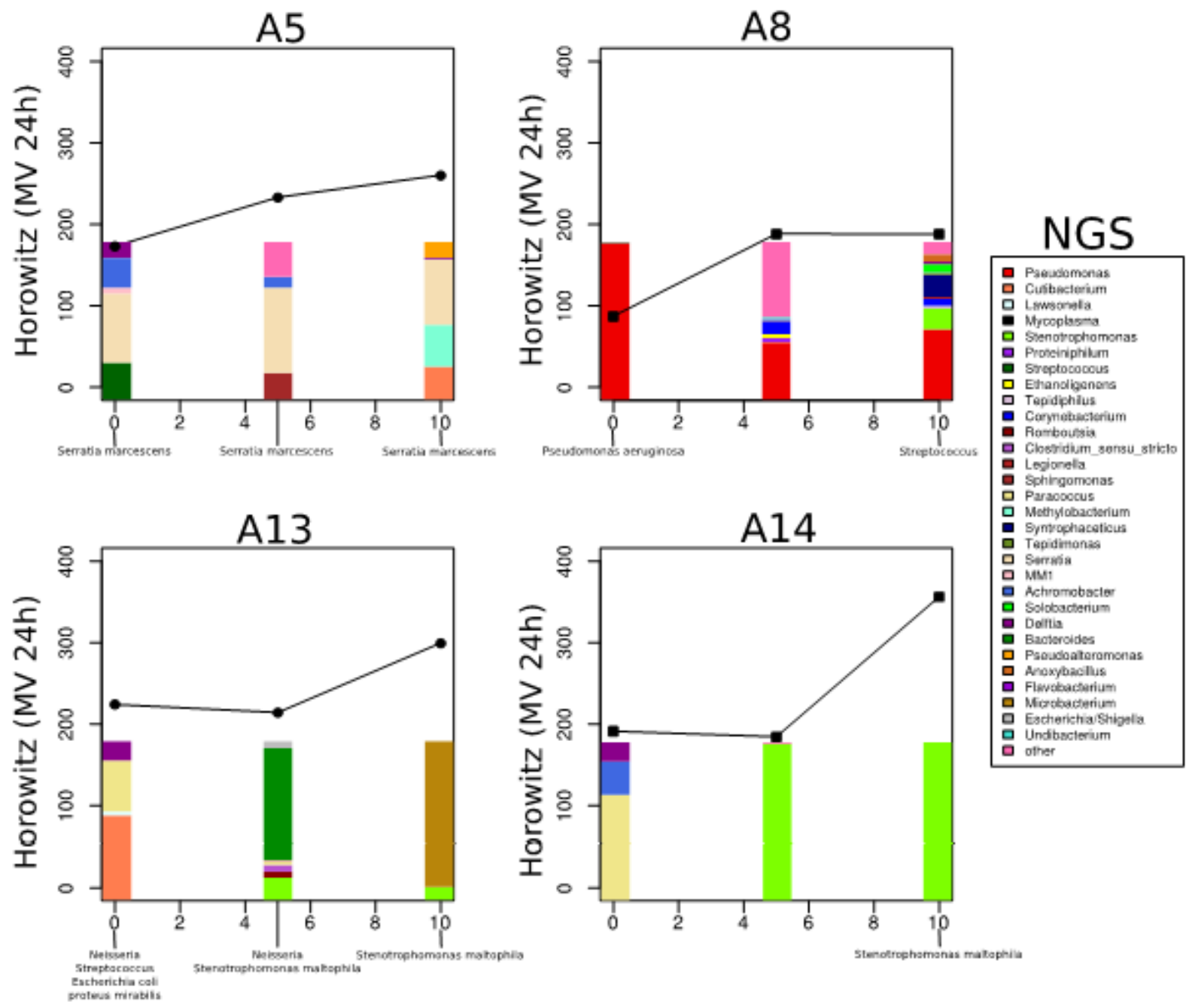

\section{Figure 4}

Comparison of NGS microbiota screening and conventional culture during the time course in four exemplary patients. Time on the $\mathrm{x}$-axis is in days and start at the admission to the ICU. Only the most abundant genera are displayed in the bar plot as relative abundance. The rest were concatenated in the group named "other". All bars have the same height and represent $100 \%$ of the microbiota. The Horowitz index was plotted as a circle for patients with long term survival (A5 and A13) and a square for patients who deceased after the study (A8 and A14). Abbreviations: NGS, next generation sequencing, ICU, intensive care unit; OTU, operational taxonomic units; PCT, Procalcitonin; SOFA, Sepsis-related organ failure assessment (-score); BMI, body mass index; CRP, C-reactive protein; MV, mean value.

\section{Supplementary Files}

This is a list of supplementary files associated with this preprint. Click to download.

- CASTRASuppl.Fig.125.10.2019.docx 
- SupplementaryFigure1Legend.docx

Page 19/19 